\title{
Effect of Tween 80 and Acetone on the Secretion, Structure and Antioxidant Activities of Exopolysaccharides from Lentinus tigrinus
}

\author{
Peixin $\mathrm{He}^{1,2}$, Shuangshuang $W u^{1}$, Lige $\operatorname{Pan}^{1}$, Siwen Sun ${ }^{1}$, Duobin Mao ${ }^{1,2}$ and \\ Chunping $X u^{1,2 *}$ \\ ${ }^{1}$ School of Food and Biological Engineering, Zhengzhou University of Light Industry, \\ Zhengzhou 450001, PR China \\ ${ }^{2}$ Collaborative Innovation Center of Food Production and Safety, Henan Province, \\ Zhengzhou 450001, PR China
}

Received: April 14, 2015

Accepted: April 14, 2016

\begin{abstract}
Summary
In this study, the effects of the addition of Tween 80 and acetone on secretion, structure and antioxidant activities of Lentinus tigrinus exopolysaccharides (EPS) were investigated. It was found that Tween 80 and acetone displayed a stimulatory effect on EPS secretion. The EPS obtained by the addition of Tween 80 (EPS-T), acetone (EPS-A) and control (EPS-C) were purified by Sepharose CL-6B gel filtration chromatography and molecular mass of purified fractions was estimated to be 22.1, 137 and $12 \mathrm{kDa}$, respectively. Monosaccharide composition analysis indicated that EPS-T, EPS-A and EPS-C were mainly composed of glucose and mannose. Congo Red test indicated that EPS-T and EPS-A had a highly ordered conformation of triple helix, while EPS-C had a random coil conformation. Furthermore, EPS-A exhibited higher DPPH scavenging and antiproliferative activities than EPS-C and EPS-T, which might be attributed to the molecular mass.
\end{abstract}

Key words: exopolysaccharides, Lentinus tigrinus, molecular mass, molecular conformation, antiproliferative activity

\section{Introduction}

Intracellular polysaccharides (IPS) extracted from mycelia and fruiting bodies of mushrooms and exopolysaccharides (EPS) from submerged culture broth have been widely studied and exploited for their multiple pharmacological activities $(1,2)$. Production of EPS in submerged culture has the potential advantages of saving time and space, as well as the possibility of industrial production (3).

Lentinus tigrinus is a white-rot fungus naturally occurring on rotten hardwood during spring and summer in China (4). It is an edible and medicinal mushroom as it possesses high nutritive and health value with high quan- tity of amino acids, few sugars and low calories (5). The natural compounds from this mushroom, such as phenolics, are proved to have antioxidant activity (6). However, there are no reports on the bioactivity of polysaccharides from L. tigrinus.

Stimulating agents, such as surfactants, vegetable oil and organic solvents are reported to mediate cell permeabilization and thus enhance the secretion of intracellular target products (7). On the other hand, the surfactant and organic solvents are inexpensive and may be removed by simple evaporation. Hence, they are used frequently and effectively for cell permeabilization $(8,9)$. The representative surfactants that enhance cell membrane permeability 
are Tween 40, Tween 60 and Tween 80, while commonly used organic solvents are toluene, chloroform, ethanol, ether and dimethyl sulfoxide $(7,9)$.

Stimulating agents are rarely reported to be used to enhance the permeability of fungi and improve the productivity of specific metabolites (10). Plant oil and fatty acids have been used in fungal fermentations to accelerate and enhance the mycelial growth and EPS secretion (11). However, the effect of various surfactants and organic solvents on the secretion and structure of EPS in submerged culture of medicinal fungi was not reported. In this study, the effects of several surfactants and organic solvents in an attempt to enhance EPS secretion in submerged culture of L. tigrinus were compared. Tween 80 and acetone were selected to enhance EPS secretion. After isolation and purification, the molecular mass and chemical characteristics of EPS obtained with the addition of Tween 80 (EPS-T), acetone (EPS-A) and control (EPS-C, i.e. without the addition of an agent) were determined. Finally, antioxidant and antiproliferative activity of the EPS were also evaluated and compared.

\section{Materials and Methods}

\section{Mushroom isolation and growth conditions}

Lentinus tigrinus was obtained by tissue isolation method from naturally occurring fruiting body and identified by Prof Xinsheng He of Southwest University of Science and Technology, Mianyang, PR China. This strain was maintained on potato dextrose agar (PDA) slant (potato $200 \mathrm{~g}$, glucose $20 \mathrm{~g}$, agar $15 \mathrm{~g}$ and deionized water $1000 \mathrm{~mL}$ ) at the Henan Province Microbiological Culture Collection Center (HPMCC no. 35481696) (12). The strain was transferred into the seed medium $(30 \mathrm{~g} / \mathrm{L}$ of glucose and $3 \mathrm{~g} / \mathrm{L}$ of yeast extract) by punching out a disc $(5 \mathrm{~mm})$ from the plate culture. The seed culture was grown in a 250 -mL flask containing $50 \mathrm{~mL}$ of the medium at $26^{\circ} \mathrm{C}$ on a rotary shaker incubator $(210 \times g)$ for four days.

\section{Flask culture and the treatment with surfactant and organic solvent}

The flask culture experiments were performed in a 500-mL Erlenmeyer flask containing $150 \mathrm{~mL}$ of the culture medium (lactose $60 \mathrm{~g}$, yeast extract $4 \mathrm{~g}$ and distilled water $1000 \mathrm{~mL} ; \mathrm{pH}=5.0)$ at $26^{\circ} \mathrm{C}$ for 12 days after inoculation with $4 \%$ (by volume) of the seed culture. To find a suitable surfactant and organic solvents for EPS secretion by $L$. tigrinus, various surfactants (Tween 40, Tween 60 and Tween 80 from Sigma-Aldrich, St. Louis, MO, USA) and organic solvents (chloroform, ethanol, DMSO and acetone from Sigma Chemical Corp., St. Louis, MO, USA) were added at a volume fraction of $0.2 \%$ to the broth after 8 days of cultivation and continued to cultivate up to 12 days. All determinations were done in triplicate to ensure the observed trends were reproducible.

\section{Mycelial dry mass and EPS determination}

The mycelial pellets and culture broth were separated by filtration. The mycelial dry mass was measured after repeated washing and drying of the pellets. Crude
EPS were obtained after concentration, ethanol precipitation and centrifugation. The total sugar content was determined by the phenol-sulfuric acid method by measuring the absorbance at $490 \mathrm{~nm}$ using glucose as the standard (13).

\section{Purification and molecular characterization of EPS}

The crude EPS were purified by deproteinization, dialysis and gel filtration chromatography on Sepharose CL-6B (Sigma Chemical Corp.) (13). Protein concentration was determined by Bradford (14) using bovine serum albumin (BSA; Sigma-Aldrich) as the standard. After hydrolyzation, reduction and acetylation, the alditol acetates of refined EPS were analyzed by GC-MS (model Star 3600 CX; Varian Co., Lexington, MA, USA). The infrared spectrum of the refined EPS was measured through a Bruker Tensor 27 Fourier transform infrared (FTIR) spectrometer (Bruker Optics, Ettlingen, Germany) using $\mathrm{KBr}$ disc technique (15). The EPS molecular mass in the solution was determined by size-exclusion chromatography using a Sepharose CL-6B column (Sigma Chemical Corp.). Standard dextran series (D150, 70, 40, and 10 kDa; Sigma Chemical Corp.) were used for determination of molecular mass of EPS. The separation was carried out at $22{ }^{\circ} \mathrm{C}$ at a flow rate of $0.6 \mathrm{~mL} / \mathrm{min}$. The calibration curve of partition coefficient $\left(K_{\mathrm{av}}\right)$ vs. log molecular mass was made, in which $K_{\mathrm{av}}$ was calculated using the following equation:

$$
K_{\mathrm{av}}=\left(V_{\mathrm{e}}-V_{0}\right) /\left(V_{\mathrm{t}}-V_{0}\right)
$$

where $V_{\mathrm{e}}$ is the elution volume $(\mathrm{mL}), V_{0}$ is the column void volume $(\mathrm{mL})$ and $V_{\mathrm{t}}$ is the total column bed volume $(\mathrm{mL})$.

\section{Helix-coil transition assay}

The conformational structure of the EPS in solution was determined by the addition of Congo Red, which forms complexes with EPS. The transition from a triple helix arrangement to the single-stranded conformation was determined by measuring the absorbance at $\lambda_{\max }=$ 400-800 nm of Congo Red and EPS solution after the addition of 0 to $0.5 \mathrm{~mol} / \mathrm{L}$ of $\mathrm{NaOH}$ with the UV-Vis spectrophotometer (TU-1800; Beijing Purkinje General Instrument Co. Ltd., Beijing, PR China) according to our previous report (16).

\section{Antioxidant activity assays}

The scavenging activity of hydroxyl and DPPH radicals was evaluated to determine the antioxidant activity of the samples according to our previous report (17). In the assays, 1,1-diphenyl-2-picrylhydrazyl (DPPH; Sigma Chemical Co.) and phenanthroline (Sigma Chemical Co.) were used.

\section{Antiproliferative activity assay}

The antiproliferative activity of EPS was measured using the previously reported MTT assay (13). Human hepatoma (Hep G2) and human osteoblast-like (MG-63) cells obtained from Cancer Institute of the Fourth Military Medical University (Xi'an, PR China) were employed and the concentrations of EPS of 25, 50, 100, 200 and $400 \mu \mathrm{g} /$ $\mathrm{mL}$ were tested in this assay. 


\section{Statistical analysis}

The results were reported as mean value \pm standard deviation (S.D.). One-way analysis of variance (ANOVA) was carried out for the comparison of mean values, and those at $\mathrm{p}<0.05$ were considered statistically significant.

\section{Results and Discussion}

\section{Effect of surfactants and organic solvents on EPS secretion}

Among the tested surfactants and organic solvents, Tween 40 and chloroform enhanced the mycelial growth (Table 1). However, the EPS secretion was improved by 22.4 or $107.2 \%$ when Tween 80 or acetone was used, respectively. Similar results were observed by Liu and $\mathrm{Wu}$ (18), who found that Tween 80 had a remarkable promoting effect on EPS secretion by Cordyceps sinensis Cs-HK1,

Table 1. Effect of surfactants and organic solvents on mycelial growth and exopolysaccharide (EPS) production by Lentinus tigrinus in shake flask cultures

\begin{tabular}{ccc}
\hline & $\gamma($ mycelium $) /(\mathrm{g} / \mathrm{L})$ & $\gamma($ EPS $) /(\mathrm{g} / \mathrm{L})$ \\
\hline Control & $9.47 \pm 0.05$ & $3.35 \pm 0.09$ \\
Surfactant & & \\
Tween 40 & $10.40 \pm 0.12$ & $3.07 \pm 0.06$ \\
Tween 60 & $4.80 \pm 0.01$ & $2.38 \pm 0.09$ \\
Tween 80 & $5.70 \pm 0.02$ & $4.10 \pm 0.27$ \\
Organic solvent & & \\
Chloroform & $13.40 \pm 0.03$ & $5.15 \pm 0.74$ \\
Ethanol & $8.67 \pm 0.02$ & $5.36 \pm 0.11$ \\
DMSO & $8.30 \pm 0.01$ & $3.29 \pm 0.38$ \\
Acetone & $7.80 \pm 0.01$ & $6.94 \pm 0.09$ \\
\hline
\end{tabular}

Fermentations were carried out for 12 days at $26^{\circ} \mathrm{C}$. The surfactants and organic solvents were added to the basal medium at a volume fraction of $0.2 \%$ after 8 days of cultivation. Values are mean \pm S.D. of triplicate determinations increasing the EPS yield more than twofold at the mass per volume ratio of $1.5 \%$. It was interesting to find that Tween 80 and acetone had negative effects on the mycelial biomass, which suggested that they had toxic effect on Lentinus tigrinus. The results indicated that the surfactants and organic solvents could modify the composition of the mycelial cell membrane, and thus change the permeability of L. tigrinus mycelia and enhance the EPS secretion (19). Hence, the EPS with the higher molecular mass could be secreted when surfactants and organic solvents are added to the culture medium.

\section{Comparison of molecular and structural properties of EPS}

Fig. 1a shows the elution profile of EPS obtained by the addition of Tween 80 (EPS-T) or acetone (EPS-A), and the control (without the addition of stimulating agents (EPS-C)). FTIR spectra of the three EPS types are shown in Fig. 1b. All samples showed a broad characteristic peak at approx. $3200 \mathrm{~cm}^{-1}$ typical of $-\mathrm{OH}$ groups, as well as a weak C-H band at approx. $2930 \mathrm{~cm}^{-1}$. The characteristic band at approx. 1599-1642 $\mathrm{cm}^{-1}$ could be correlated with the stretching vibration of the carbonyl group (20). A small peak at approx. $1403-1414 \mathrm{~cm}^{-1}$ assigned to amino groups was also observed in the FTIR spectra. Bands at approx. 1026-1094 $\mathrm{cm}^{-1}$ suggested the presence of C-O type of linkage (21). The results suggest that the addition of Tween 80 and acetone does not affect the functional groups of the EPS.

The molecular masses of EPS-T, EPS-A and EPS-C were 22.1, 137 and $12 \mathrm{kDa}$, respectively (Fig. 2). The EPS-T and EPS-A had higher molecular masses than EPS-C, which indicated that surfactants and organic solvents interacted with the phospholipid bilayer membranes and decreased the cell membrane permeability. The monomer composition of the three EPS types determined by GC-MS analysis is shown in Table 2. Results indicate that glucose and mannose are the major monosaccharides in all EPSs although their fraction is markedly different, while Tween 80 and acetone could alter the internal metabolism by affecting the enzymatic synthesis (7).
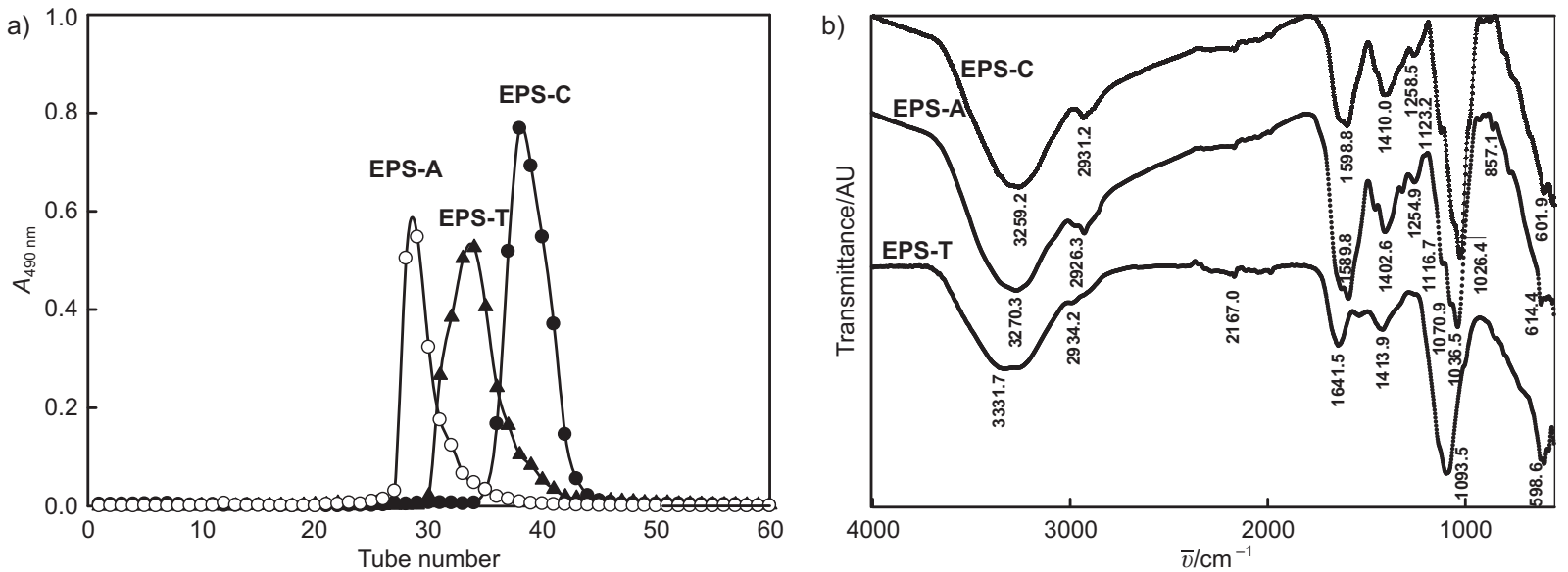

Fig. 1. The results of: a) size-exclusion chromatography in Sepharose CL-6B column, and b) the FTIR spectra of the exopolysaccharides (EPS) produced by submerged culture of Lentinus tigrinus using Tween 80 (EPS-T) and acetone (EPS-A), in comparison with control (EPS-C). $V($ sample $)=5 \mathrm{~mL}$ 


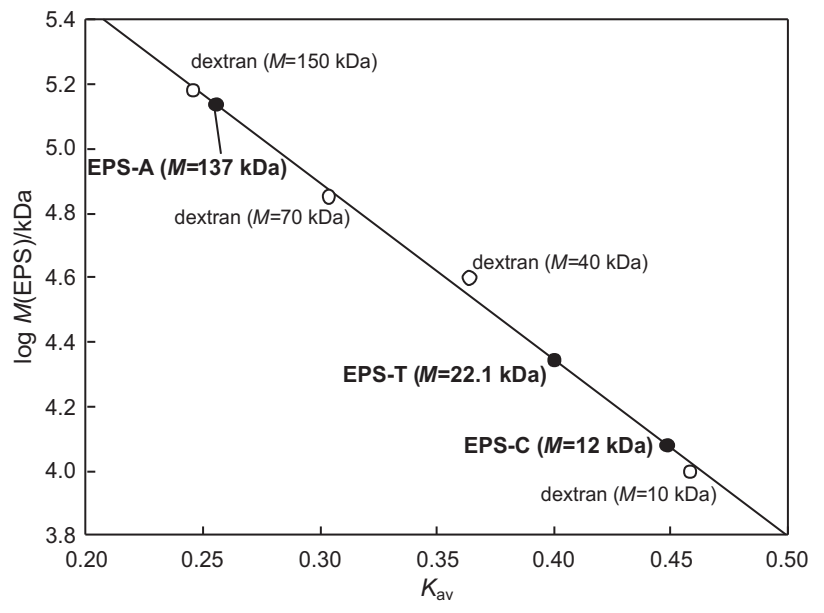

Fig. 2. Molecular mass of the purified exopolysaccharides (EPS, closed circles) produced by submerged culture of Lentinus tigrinus using Tween 80 (EPS-T) and acetone (EPS-A), in comparison with control without the addition of stimulating agents (EPS-C), on Sepharose CL-6B column. Elutes were analyzed by measuring the absorbance at $490 \mathrm{~nm}\left(A_{490} \mathrm{~nm}\right)$. Open circles represent different dextrans used as control. $K_{\mathrm{av}}=$ partition coefficient

Table 2. Carbohydrate composition of the purified Lentinus tigrinus exopolysaccharides (EPS) produced with Tween 80 or acetone

\begin{tabular}{|c|c|c|c|}
\hline$w($ carbohydrate) & & EPS & \\
\hline$\%$ & Control & Tween 80 & Acetone \\
\hline Xylose & $3.54 \pm 0.05$ & $0.00 \pm 0.00$ & $2.81 \pm 0.04$ \\
\hline Mannose & $60.11 \pm 2.17$ & $30.94 \pm 2.01$ & $45.36 \pm 1.02$ \\
\hline Rhamnose & $0.00 \pm 0.00$ & $0.00 \pm 0.00$ & $0.30 \pm 0.01$ \\
\hline Galactose & $10.57 \pm 0.07$ & $6.61 \pm 0.09$ & $6.20 \pm 0.07$ \\
\hline Glucose & $24.53 \pm 1.83$ & $58.88 \pm 2.08$ & $43.82 \pm 1.11$ \\
\hline Trehalose & $0.00 \pm 0.00$ & $3.57 \pm 0.02$ & $0.00 \pm 0.00$ \\
\hline Ribose & $1.25 \pm 0.02$ & $0.00 \pm 0.00$ & $1.51 \pm 0.03$ \\
\hline
\end{tabular}

Congo Red test can rapidly detect the polysaccharide conformation (22). The $\lambda_{\max }$ of all complexes of Congo Red with EPS is shown in Fig. 3. The addition of EPS-C (in a complex with Congo Red) did not cause any notable change in the $\lambda_{\max }$ (Fig. 3a) with the increase of $\mathrm{NaOH}$ concentration, as compared to that of Congo Red alone. This showed that the EPS-C had a random coil conformation in the aqueous solution. However, at low concentrations (0-0.2 mol/L), the $\lambda_{\max }$ shift to a longer wavelength can be observed (Figs. $3 b$ and c). When the concentration of $\mathrm{NaOH}$ was increased to more than $0.2 \mathrm{~mol} / \mathrm{L}$, the $\lambda_{\max }$ slowly dropped. This indicated that EPS-A and EPS-T had a highly ordered conformation of triple helix, which remained stable even under strong alkaline conditions, as reported previously (22).

\section{Antioxidant and antiproliferative activities}

In vitro antioxidant activities of three types of EPS from L. tigrinus were evaluated using hydroxyl and DPPH radical scavenging assays. The EPS showed strong hydroxyl radical scavenging capacities in a concentration-dependent manner; however, they were not notably different (Fig. 4a). The DPPH scavenging rates of the tested EPS (Fig. 4b) confirmed that the DPPH scavenging activity of EPS-A was better than those of EPS-C and EPS-T, reaching a maximum of $63.0 \%$ at mass concentration of 8 $\mathrm{mg} / \mathrm{mL}$. The stronger antioxidant activity of EPS-A than those of EPS-C and EPS-T may be attributed to their difference in molecular mass $(23,24)$.

The growth inhibitory effects of the polysaccharides (in the concentration of $50-400 \mu \mathrm{g} / \mathrm{mL}$ ) against Hep G2 and MG-63 cells were examined. The antitumour activity against Hep G2 increased significantly (Fig. 4c) with the corresponding increase in the concentration of EPS $(p<0.05)$. EPS-A exhibited higher antiproliferative activity than EPS-C and EPS-T. Similarly to Hep G2, the antitumour activities against MG-63 were also concentration dependent (Fig. 4d). EPS-A also had higher antiproliferative activity than other EPS samples. The results indicated that the molecular mass may be the main factor determining the antiproliferative activity of fungal EPS. Zhang et al. (25) found that the EPS from the Pleurotus tuber-regium mycelia exhibited higher antitumour activity in the molecular mass range of $400-800 \mathrm{kDa}$ than other fractions with molecular mass below $50 \mathrm{kDa}$ in vivo and in vitro.

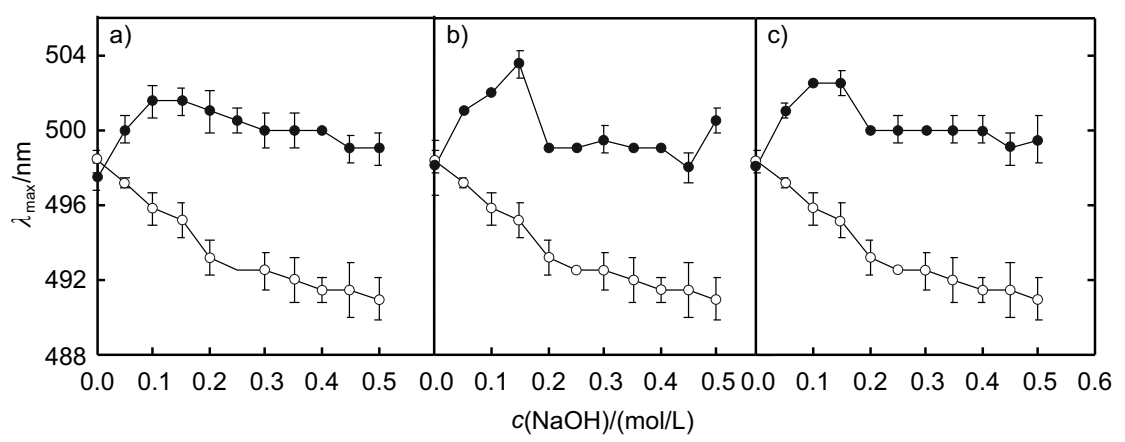

Fig. 3. The results of Congo Red test of exopolysaccharides (EPS, closed circles) produced by submerged culture of L. tigrinus using: a) Tween 80 (EPS-T), b) acetone (EPS-A), and c) control without the addition of stimulating agents (EPS-C), presented as wavelength of absorption maxima of the complex of Congo Red with polysaccharide at various concentrations of $\mathrm{NaOH}$. Congo Red in $\mathrm{NaOH}$ and dextran served as standards (open circles) 

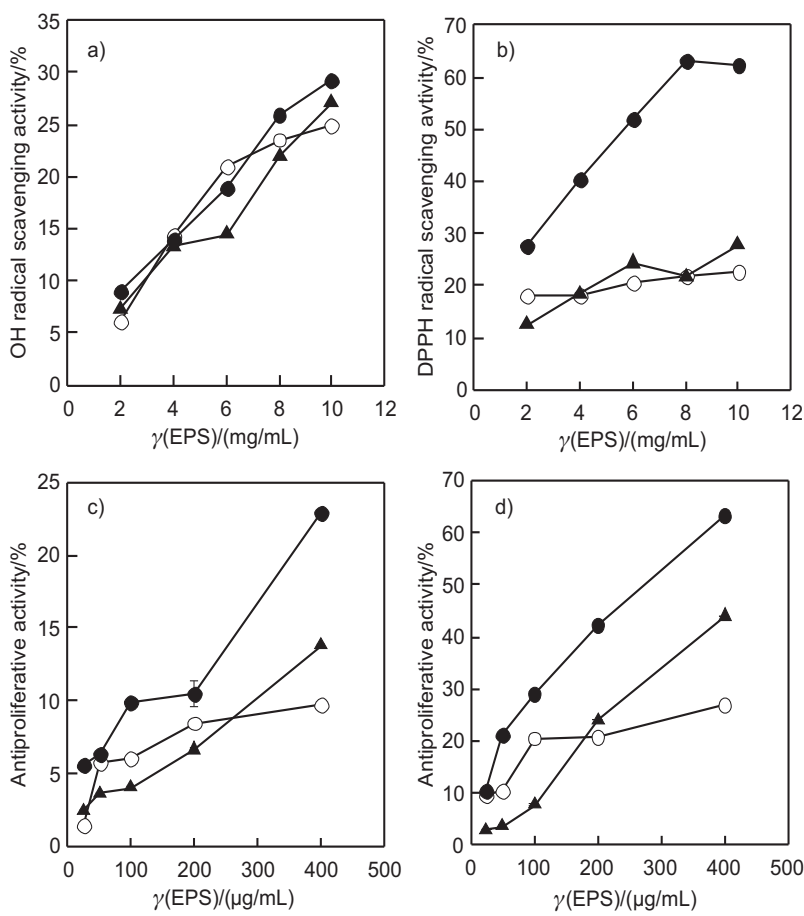

Fig. 4. Bioactivity of exopolysaccharides (EPS) produced by submerged culture of Lentinus tigrinus using Tween 80 (EPS-T, $\Delta)$ and acetone (EPS-A, $\bullet$ ). The results represent mean value \pm S.D. $(N=3)$ : a) $\mathrm{OH}$ and b) DPPH radical scavenging activity and antiproliferative activity against: c) Hep G2, and d) MG-63 cells, in comparison with control (EPS-C, O). Cells were grown on 96-well plates and allowed to attach for $16 \mathrm{~h}$, and cell viability was determined by MTT assay

\section{Conclusions}

The effects of surfactants and organic solvents on secretion, characterization and bioactivity of exopolysaccharides (EPS) from Lentinus tigrinus were investigated. The results demonstrated that Tween 80 and acetone could enhance EPS secretion but they interfere with the mycelial growth. The molecular characterization and bioactivity of EPS treated with Tween 80 (EPS-T) and acetone (EPS-A) were compared. EPS-A showed stronger DPPH radical scavenging and antiproliferative activities, which might be related to its molecular mass. The stimulation of EPS biosynthesis in the fungal cells and improvement of various biological activities need further investigation.

\section{References}

1. Ünyayar A, Demirbilek M, Turkoglu M, Celik A, Mazmanci MA, Erkurt EA, et al. Evaluation of cytotoxic and mutagenic effects of Coriolus versicolor and Funalia trogii extracts on mammalian cells. Drug Chem Toxicol. 2006;29:69-83. http://dx.doi.org/10.1080/01480540500408655

2. Wasser SP. Medicinal mushroom science: history, current status, future trends, and unsolved problems. Int J Med Mushrooms. 2010;12:1-16.

http://dx.doi.org/10.1615/IntJMedMushr.v12.i1.10

3. Yang FC, Huang HC, Yang MJ. The influence of environmental conditions on the mycelial growth of Antrodia cinnamomea in submerged cultures. Enzyme Microb Tech. 2003;33: 395-402.

http://dx.doi.org/10.1016/S0141-0229(03)00136-4
4. Dulay RMR, Kalaw SP, Reyes RG, Cabrera EC, Alfonso NF. Optimization of culture conditions for mycelial growth and basidiocarp production of Lentinus tigrinus (Bull.) Fr., a new record of domesticated wild edible mushroom in the Philippines. Philipp Agric Scientist. 2012;95:278-85.

5. Lechner BE, Papinutti VL. Production of lignocellulosic enzymes during growth and fruiting of the edible fungus Lentinus tigrinus on wheat straw. Process Biochem. 2006;41: 594-8. http://dx.doi.org/10.1016/j.procbio.2005.08.004

6. Reis FS, Pereira E, Barros L, Sousa MJ, Martins A, Ferreira ICFR. Biomolecule profiles in inedible wild mushrooms with antioxidant value. Molecules. 2011;16:4328-38. http://dx.doi.org/10.3390/molecules16064328

7. De León A, García B, Barba de la Rosa AP, Villaseñor F, Estrada A, López-Revilla R. Periplasmic penicillin G acylase activity in recombinant Escherichia coli cells permeabilized with organic solvents. Process Biochem. 2003;39:301-5. http://dx.doi.org/10.1016/S0032-9592(03)00079-7

8. Choi KO, Song SH, Yoo YJ. Permeabilization of Ochrobactrum anthropi SY509 cells with organic solvents for whole cell biocatalyst. Biotechnol Bioprocess Eng. 2004;9:147-50. http://dx.doi.org/10.1007/BF02942284

9. De Smet MJ, Kingma J, Witholt B. The effect of toluene on the structure and permeability of the outer and cytoplasmic membranes of Escherichia coli. BBA - Biomembranes. 1978; 506:64-80.

http://dx.doi.org/10.1016/0005-2736(78)90435-2

10. Reese ET, Maguire A. Surfactants as stimulants of enzyme production by microorganisms. Appl Microbiol. 1969;17: 242-5.

11. Stasinopoulos SJ, Seviour RJ. Stimulation of exopolysaccharide production in the fungus Acremonium persicinum with fatty acids. Biotechnol Bioeng. 1990;36:778-82. http://dx.doi.org/10.1002/bit.260360804

12. Park JP, Kim SW, Hwang HJ, Yun JW. Optimization of submerged culture conditions for the mycelial growth and exobiopolymer production by Cordyceps militaris. Lett Appl Microbiol. 2001;33:76-81. http://dx.doi.org/10.1046/j.1472-765X.2001.00950.x

13. He P, Geng L, Wang Z, Mao D, Wang J, Xu C. Fermentation optimization, characterization and bioactivity of exopolysaccharides from Funalia trogii. Carbohydr Polym. 2012;89:1723. http://dx.doi.org/10.1016/j.carbpol.2012.01.093

14. Bradford MM. A rapid and sensitive method for the quantitation of microgram quantities of protein utilizing the principle of protein-dye binding. Anal Biochem. 1976;72:248-54. http://dx.doi.org/10.1016/0003-2697(76)90527-3

15. Ma Y, Mao D, Geng L, Wang Z, Xu C. Production, fractionation, characterization of extracellular polysaccharide from a newly isolated Trametes gibbosa and its hypoglycemic activity. Carbohydr Polym. 2013;96:460-5. http://dx.doi.org/10.1016/j.carbpol.2013.04.019

16. Cao J, Zhang HJ, Xu CP. Culture characterization of exopolysaccharides with antioxidant activity produced by Pycnoporus sanguineus in stirred-tank and airlift reactors. J Taiwan Inst Chem Eng. 2014;45:2075-80. http://dx.doi.org/10.1016/j.jtice.2014.05.005

17. Zheng JQ, Mao XJ, Geng LJ, Yang GM, Xu CP. Production optimization, preliminary characterization and bioactivity of exopolysaccharides from Incutis tamaricis (Pat.) Fiasson \& Niemela. J Taiwan Inst Chem Eng. 2014;45:725-33. http://dx.doi.org/10.1016/j.jtice.2013.08.006

18. Liu YS, Wu JY. Effects of Tween 80 and $\mathrm{pH}$ on mycelial pellets and exopolysaccharide production in liquid culture of a medicinal fungus. J Ind Microbiol Biotechnol. 2012;39:623-8. http://dx.doi.org/10.1007/s10295-011-1066-9 
19. Zhang BB, Cheung PCK. A mechanistic study of the enhancing effect of Tween 80 on the mycelial growth and exopolysaccharide production by Pleurotus tuber-regium. Bioresour Technol. 2011;102:8323-6. http://dx.doi.org/10.1016/j.biortech.2011.06.021

20. Wang JL, Zhang J, Zhao BT, Wang XF, Wu YQ, Yao J. A comparison study on microwave-assisted extraction of Potentilla anserine L. polysaccharides with conventional method: molecule weight and antioxidant-activities evaluation. Carbohydr Polym. 2012;80:84-93. http://dx.doi.org/10.1016/j.carbpol.2009.10.073

21. Fan Y, He XJ, Zhou S, Luo A, He T, Chun Z. Composition analysis and antioxidant activity of polysaccharide from Dendrobium denneanum. Int J Biol Macromol. 2009;45:16973. http://dx.doi.org/10.1016/j.ijbiomac.2009.04.019

22. Bao XF, Zhen Y, Ruan L, Fang JN. Purification, characterization, and modification of $\mathrm{T}$ lymphocyte-stimulating polysaccharide from spores of Ganoderma lucidum. Chem Pharm
Bull. 2002;50:623-9.

http://dx.doi.org/10.1248/cpb.50.623

23. Nie Y, Jiang H, Su Y, Zhu C, Li J, Wen F. Purification, composition analysis and antioxidant activity of different polysaccharides from the fruiting bodies of Pholiota adipose. Afr J Biotechnol. 2012;11:12885-94.

http://dx.doi.org/10.5897/AJB11.3117

24. Wang J, Zhang QB, Zhang ZS, Song HF, Li PC. Potential antioxidant and anticoagulant capacity of low molecular weight fucoidan fractions extracted from Laminaria japonica. Int J Biol Macromol. 2010;46:6-12.

http://dx.doi.org/10.1016/j.ijbiomac.2009.10.015

25. Zhang M, Zhang L, Cheung PCK, Ooi VEC. Molecular weight and anti-tumor activity of the water-soluble polysaccharides isolated by hot water and ultra-sonic treatment from the sclerotia and mycelia of Pleurotus tuber-regium. Carbohydr Polym. 2004;56:123-8.

http://dx.doi.org/10.1016/j.carbpol.2004.01.005 